\title{
Modélisation continue tridimensionnelle du comportement mécanique d'un mur en Terre Armée
}

\section{A. BREUGNOT ${ }^{1,2}$} L. BOUTONNIER ${ }^{1}$

${ }^{1}$ EGIS Géotechnique 1, rue du Docteur-Schweitzer 38180 Seyssins

${ }^{2}$ Laboratoire 3S-R, UMR Domaine universitaire BP 53

38041 Grenoble Cedex 9 antonin.breugnot@egis.fr luc.boutonnier@egis.fr
Cet article présente les modélisations effectuées par Egis Géotechnique dans le cadre du concours de prévisions concernant le comportement mécanique d'un ouvrage en Terre Armée. Pour évaluer à la fois les déformations de l'ouvrage ainsi que les efforts de tractions dans les armatures, une approche continue tridimensionnelle a été retenue. L'ensemble des matériaux granulaires est caractérisé par des lois élasto-plastiques avec des critères de rupture de type Mohr-Coulomb. Pour décrire le plus fidèlement possible l'interaction sol-structure au niveau des renforcements, ceux-ci sont modélisés individuellement par des éléments structuraux avec une interface dont le comportement est élastique parfaitement plastique. Un soin particulier a été apporté à l'initialisation des contraintes pour essayer de prendre en compte correctement l'effet du compactage sur le coefficient de pression des terres au repos $\mathrm{K}_{0}$ dans le domaine surconsolidé. Les prévisions obtenues numériquement sont présentées et confrontées aux résultats obtenus expérimentalement. Dans un deuxième temps, la sensibilité des différents paramètres, dont la détermination a fait l'objet d'hypothèses importantes, est également évaluée avec une étude paramétrique.

Mots-clés : mur en Terre Armée, interaction sol/structure, modélisation numérique, initialisation des contraintes.

This article presents the models and the assumptions retained by Egis Géotechnique within the framework of the reinforced earth wall benchmark study. To estimate structural deformations as well as the tensile forces in the steel reinforcements, a three dimensional continuum approach is proposed. Granular materials are characterised by elasto-plastic laws with a MohrCoulomb failure criterion. In order to accurately simulate soil/ structure interactions, the reinforcements are individually modelled by structural elements with an interface that is elastic perfectly plastic. The compaction effect to the $\mathrm{K}_{0}$ coefficient, in the over-consolidated domain, is taken into account during stress initialisation. The model predictions obtained with no parameter fitting are presented and compared with the experimentally obtained results. The second part evaluates the influence of main parameters using a parametric study. 


\section{Introduction}

Les ouvrages de soutènement en sol renforcé de type Terre Armée présentent un nombre important d’inclusions linéaires permettant d'obtenir des caractéristiques mécaniques apparentes élevées.

Les méthodes de dimensionnement actuelles relèvent plus d'une analyse à la rupture en force (Norme NF P94-220-0) que d'une analyse en déformation des ouvrages considérés.

La participation d'Egis Géotechnique au concours de prévisions du comportement d'un mur en Terre Armée s'est faite dans le but d'améliorer les modèles de calcul en déformations, via des outils de simulation, en confrontant les résultats issus des modèles numériques à ceux obtenus expérimentalement.

La modélisation des essais de chargement du plot expérimental est menée via une approche continue tridimensionnelle permettant de modéliser individuellement chaque élément de renforcement. Une attention particulière a été portée à la fine représentation cles interfaces entre le sol et les armatures métalliques ainsi qu'à l'initialisation des contraintes pour essayer de prendre en compte correctement l'effet du compactage sur l'état de contrainte induit dans l'ouvrage par le mode de construction.

\section{2}

\section{Description de l'ourrage modélisé}

L'ouvrage d'étude (Fig. 1) a été construit dans une fosse d'essais, constituée en majeure partie par le sol naturel, et est constitué de cuatre matériaux granulaires, à savoir : le sol de remblai sur une hauteur de $3,5 \mathrm{~m}$, une sous-couche d'épaisseur $30 \mathrm{~cm}$, le ballast sur $30 \mathrm{~cm}$ également et enfin la fosse utilisée comme condition limite au modèle de l'ouvrage.

Le corps du remblai est renforcé par cinclits de dix armatures métalliques, et la stabilité superficielle des matériaux, au niveau du parement, est assurée par un ensemble formé d'une quinzaine d'écailles en béton.

\section{3}

\section{Principes de modélisation}

A l'exception de la poutre de chargement, l'ensemble des couches de matériau et tous les éléments de renforcement sont représentés et modélisés par une approche de mécanique des milieux continus.
Des modèles différents sont cependant utilisés en fonction de la géométrie de l'objet modélisé. Ainsi les milieux granulaires sont modélisés par des éléments continus volumiques, tandis que les renforcements (armatures métalliques et écailles du parement) le sont via des éléments continus linéiques ou surfaciques. La jonction entre les éléments de renforcement et le sol est assurée par des interfaces permettant de contrôler les déplacements relatifs entre ces différents constituants.

L'outil de simulation utilisé Flac3D (Itasca, 2006) est un code de calcul basé sur une application non traditionnelle de la méthode aux différences finies explicite. Sa formulation numérique est articulée autour d'une description lagrangienne du mouvement.

En simulant en trois dimensions des structures composées de sols au comportement élastoplastique, Flac3D est particulièrement adapté à la résolution de problèmes en géomécanique.

Le recours à un modèle $3 \mathrm{D}$ est motivé à la fois par le chargement localisé au centre de l'ouvrage et non réparti sur toute sa largeur, et par la nature de la répartition géométrique des armatures dans l'ouvrage. Représenter l'assemblage 3D des inclusions discrètes par des “ bandes » continues dans un modèle 2D serait une simplification dont il est difficile d'évaluer les conséquences sur la pertinence du modèle.

\section{4}

\section{Comportement des matériaux granulaires}

Les matériaux granulaires sont modélisés par des éléments volumiques et caractérisés par des lois élastoplastiques avec un critère de rupture en cisaillement de type Mohr-Coulomb.

Les paramètres du modèle sont le module d'Young E, le coefficient de Poisson $v$, l'angle de frottement interne $\varphi$, la cohésion $c$ et l'angle de dilatance $\psi$, auxquels on peut ajouter le poids volumique $\gamma$ des matériaux.

Concernant la sous-couche et le sol de remblai, la plupart des caractéristiques proviennent d'essais de caractérisation des matériaux et d'essais in situ réalisés dans le cadre du concours de prévisions. Afin de prendre en compte le caractère dilatant des matériaux constituant le corps de l'ouvrage, un angle de dilatance $\psi$ fonction de l'angle de frottement interne $\varphi$ des sols (Bolton, 1986) est finalement retenu :

$$
\psi=\varphi-30 \text { degrés }
$$

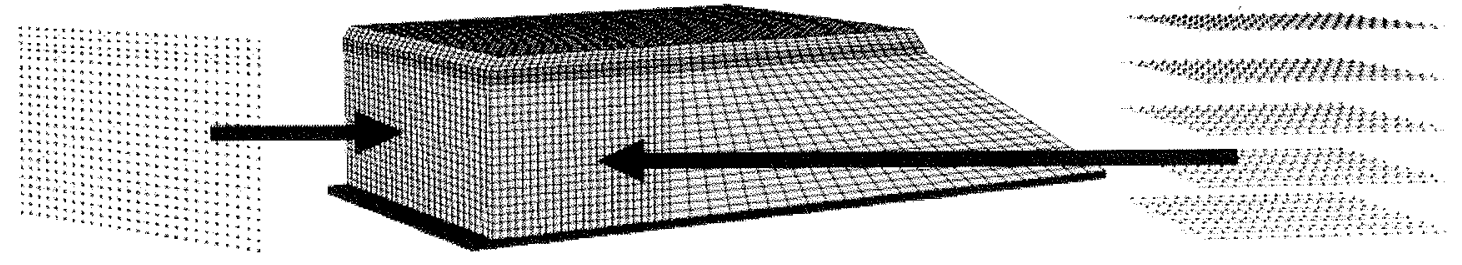

\section{a) Parement béton}

b) Massifs de sol

c) Lits d'armatures

FG. 1 Modèle numérique de l'ouvrage en Terre Armée : couches de sol (b) renforcées par les éléments du parement (a) et les lits d'armatures (c). Earth reinforced numerical model : soil layers (b) reinforced by concrete panels (a) and steel strips (c). 
Pour les autres matériaux, la dilatance n'est pas considérée.

Les caractéristiques du ballast sont extraites de la littérature et les paramètres concernant la fosse sont déterminés en considérant un matériau plus raide mais conservant le même critère de plasticité (c et $\varphi$ ) que le sol de remblai.

Les valeurs retenues pour la caractérisation des matériaux granulaires utilisés dans les différents modèles sont récapitulées dans le tableau I.

\section{5}

\section{Comportement des armatures métalliques}

Les armatures métalliques de renforcement, positionnées dans le sol de remblai, ont une section de petite dimension par rapport à la taille caractéristique du modèle. Pour limiter le nombre de nouds et par conséquent les temps de calcul, les armatures n'ont pas été maillées suivant leur section mais seulement modélisées par des éléments structurels linéiques, au comportement élastique parfaitement plastique pouvant reprendre des efforts en traction et compression mais pas en flexion.

Dans l'exploitation des résultats, on s'est toutefois assuré que les armatures ne travaillaient jamais en compression.

Les armatures à haute adhérence renforcées sont en acier galvanisé et leur comportement propre est caractérisé par une section $\mathrm{S}=2,25 \cdot 10^{-4} \mathrm{~m}^{2}$, un module $\mathrm{d}^{\prime}$ Young $\mathrm{E}=2,1.10^{5} \mathrm{MPa}$ et une masse volumique $\rho=7800 \mathrm{~kg} / \mathrm{m}^{3}$.
Dans l'ensemble des modélisations effectuées, les contraintes de traction dans les éléments de câble ne dépassent pas $55 \mathrm{MPa}$, valeur éloignée de la limite élastique de l'acier $\mathrm{R}_{e}$ qui peut, d'après la littérature, être estimée à 200-400 MPa. De ce fait, la plasticité de l'acier n'a pas été prise en compte dans le modèle.

L'interaction sol-structure au niveau du câble est modélisée par une interface caractérisée par un ressort et un palier plastique dû à une rupture en cisaillement de nature frottante et/ou cohésive. Le comportement en cisaillement de l'interface entre le sol de remblai et les câbles est décrit numériquement par une raideur de cisaillement $k_{i}$, un angle de frottement $\varphi_{i}$, une cohésion $c_{i}$, un périmètre d'interaction $p_{i}$ et une contrainte effective moyenne de confinement $\sigma_{\mathrm{m}}$ (Itasca, 2006), (Figs. 2 et 3 )

La contrainte effective de confinement $\sigma_{m}$ est calculée en chaque nœud à partir des pressions interstitielles $\mathrm{p}$ (nulles dans notre modèle) et des contraintes principales $\sigma_{1}$ et $\sigma_{2}$ dans le plan perpendiculaire à l'axe du câble :

$$
\sigma_{\mathrm{m}}=\frac{\sigma_{1}+\sigma_{2}}{2}+\mathrm{p}
$$

La contrainte effective de confinement $\sigma_{m}$ est appliquée suivant le périmètre $\mathrm{p}_{\mathrm{i}}$, supposé égal au périmètre réel de la section des armatures métalliques : $p_{i}=0,10 \mathrm{~m}$.

L'approximation numérique, consistant à considérer une contrainte effective de confinement moyenne $\sigma_{\mathrm{m}}$ pour le calcul de la résistance maximale de cisaillement au niveau de l'interface, n'est théoriquement valide que dans le cas d'une répartition isotrope des contraintes dans le sol (Fig. 4).

TABtEau Caractéristiques mécaniques des matériaux (en gras les données d'entrée du concours de prévisions, en style standard les paramètres choisis).

Mechanical characteristics of materials (Imposed values are highlighted in bold and the others are those chosen).

\begin{tabular}{|c|c|c|c|c|c|c|}
\hline Sol & $r\left(\mathrm{kN} / \mathrm{m}^{2}\right)$ & 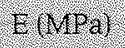 & v & $c(k \mathrm{~Pa})$ & 9 (degres) & y degres) \\
\hline Ballast & 17 & 150 & 0,2 & 0 & 45 & 0 \\
\hline Sous-couche & 23,3 & 90 & 0,3 & 10 & 36 & 6 \\
\hline Sol de remblai & 20,8 & 50 & 0,3 & 10 & 36 & 6 \\
\hline Fosse & 20,8 & 120 & 0,3 & 10 & 36 & 0 \\
\hline
\end{tabular}

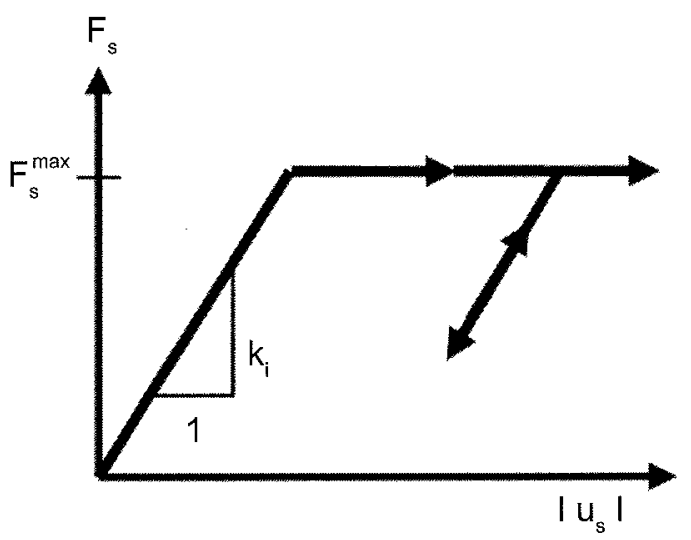

月6. 2 Force de cisaillement par mètre linéaire $F$ en fonction du déplacement relatif, en cisaillement $\left|u_{s}\right|$, entre le câble et le sol. Shear force/length $F_{s}$ versus relative shear displacement $\left|u_{s}\right|$, between the cable and the soil.

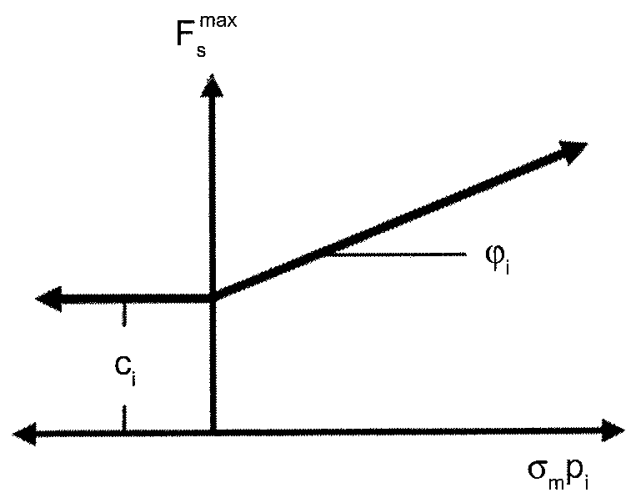

76. 3 Critère de rupture en cisaillement exprimé à partir de la force de cisaillement maximale par mètre linéaire $F_{s}{ }^{\max }$.

Shear-strength criterion defined according to the maximum shear force/length $\mathrm{F}_{\mathrm{s}}$ max 


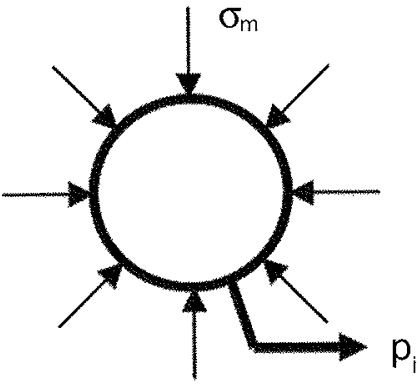

Distribution homogénéisée des contraintes autour d'une armature "numérique" à section circulaire

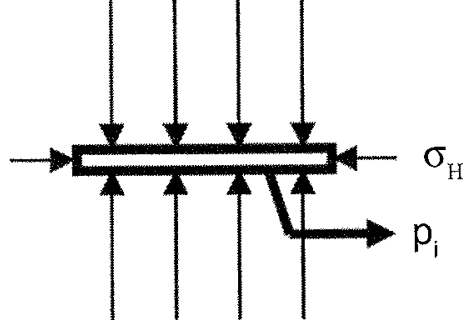

$\sigma_{v}$

Distribution "réelle" des contraintes autour d'une armature à section rectangulaire

FG. 4 Distribution des contraintes autour des armatures « numérique » et « réelle ».

Stresses distribution around ( numerical » and « real » strips.

Dans le cas contraire $\left(\sigma_{H}=K . \sigma_{V}\right.$, avec $\left.K \neq 1\right)$, le palier plastique de cisaillement est surestimé (resp. sous-estimé) pour les armatures se situant dans une zone du mur où $\sigma_{V}>\sigma_{\mathrm{H}}$ (resp. $\sigma_{\mathrm{H}}>\sigma_{\mathrm{V}}$ ), ce qui correspond plutôt à un état du sol en profondeur (resp. en surface) dans l'ouvrage.

Pour caractériser la résistance au cisaillement au niveau de l'interface, seule la composante frottante du palier plastique a été retenue. Cela revient à négliger, à long terme, l'effet de la cohésion entre le sol de remblai et l'armature. En ce qui concerne le frottement, on considère que le plan de contact sol/armature présente moins d'accroches résiduelles qu'au niveau d'un contact sol/sol. A l'interface, l'angle de frottement est estimé en ne considérant qu'une fraction de l'angle de frottement interne du sol de remblais, ce qui conduit à $\varphi_{\mathrm{i}}=27$ degrés :

$$
\tan \varphi_{i}=0,7 \times \tan \varphi_{\text {sol }}
$$

Le dernier paramètre à définir, pour caractériser le comportement des câbles et de leur interaction avec le sol, est la raideur de cisaillement d'interface $k_{i}$. Ne disposant pas de données nécessaires à l'estimation précise de ce coefficient, on a fait l'hypothèse qu'au niveau de l'armature la plus contrainte par le sol (soumis à l'effort de cisaillement résistant le plus important), le maximum de l'effort de cisaillement résistant $F_{s}$ max est mobilisé pour un déplacement, de l'armature en question, inférieur à $u_{s}=5 \mathrm{~mm}$.

La raideur de cisaillement à l'interface est alors estimée à partir de :

$$
\mathrm{k}_{\mathrm{i}}=\frac{\mathrm{F}_{\mathrm{S}}^{\max }}{\mathrm{u}_{\mathrm{S}}}
$$

$\operatorname{avec} F_{S}^{\max }=h_{\max } \times \gamma_{\text {sol }} \times \tan \left(\varphi_{i}\right) \times p_{i}$.

Cette approche fournit une valeur de $\mathrm{k}_{\mathrm{i}}$ de l'ordre de $10^{6} \mathrm{~N} / \mathrm{m}^{2}$. Ce paramètre est difficilement caractérisable sans essai d'extraction d'armature clisponible, alors que son influence peut être importante sur le comportement mécanique des armatures notamment dans de faibles gammes de déformations.

\section{Comportement du parement en béton}

Le parement du mur est constitué d'écailles embô̂tées les unes aux autres et liées par un joint ciment. Dans l'approche numérique, les écailles sont modélisées séparément, sans les joints, par des éléments de coque avec une interface caractérisant le contact entre le sol de remblai et les éléments du parement.

Par souci de simplification géométrique, les écailles cruciformes sont représentées par des éléments carrés de côté $1,5 \mathrm{~m}$.

Le comportement propre des éléments de coque est décrit par une loi élastique isotrope et l'interaction sol/structure au niveau du parement, par une interface caractérisée par deux ressorts, l'un normal au plan d'interface $\mathrm{k}_{n^{\prime}}$ l'autre dans le plan de cisaillement $\mathrm{k}_{\mathrm{s}^{\prime}}$ et par un palier plastique dû à une rupture en cisaillement de nature purement frottante.

De manière analogue au cas des armatures métalliques, l'angle de frottement à l'interface $\varphi_{i}$ entre le béton lisse et le sol peut être estimé à partir de la dégradation de l'angle de frottement interne du sol (Fig. 5).

L'interaction sol/structure au niveau du parement ne semble a priori pas déterminante sur le comportement de l'ouvrage, ainsi les ressorts d'interface $\mathrm{k}_{\mathrm{n}}$ et $\mathrm{k}_{\mathrm{s}}$ sont choisis « infiniment » rigides dans le but de limiter le comportement de l'interaction à un palier plastique défini par l'angle $\varphi_{i}$ (Tableau II).

Pour reproduire l'effet de l'enchevêtrement des écailles cruciformes, les déplacements suivant l'axe y (resp. axe z) des éléments positionnés l'un à côté de l'autre (resp. l'un au-dessus de l'autre) sont liés.

Au niveau du parement, les armatures métalliques sont liées aux écailles pour les translations, les rotations étant laissées libres. 

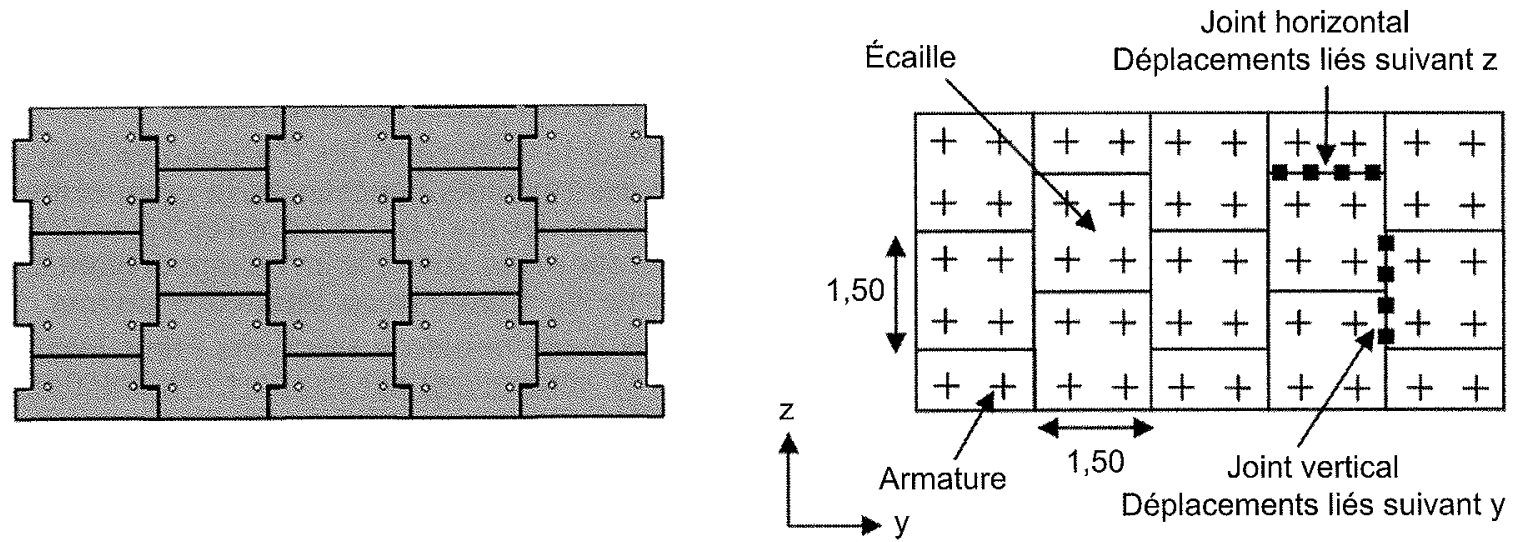

nic. 5 . Représentations schématiques des parements (( réel ) et ( numérique ),

Schemas of ( real » and « numerical ) panels.

\begin{tabular}{|c|c|c|c|c|c|c|}
\hline$T \& B O<u$ II & $\begin{array}{l}\text { Paramètres défir } \\
\text { Parameters definir }\end{array}$ & $\begin{array}{l}\text { ant le comportem } \\
\text { le behavior of concr }\end{array}$ & $\begin{array}{l}\text { des écailles c } \\
\text { panels and the }\end{array}$ & $\begin{array}{l}\text { arement et leur } \\
\text { eraction with the }\end{array}$ & $\begin{array}{l}\text { teraction avec } \\
\text { ling materials. }\end{array}$ & blai. \\
\hline 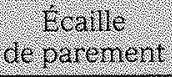 & & 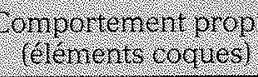 & & \multicolumn{3}{|c|}{ 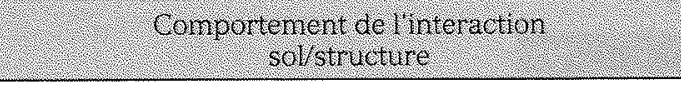 } \\
\hline Paramètres & $\begin{array}{c}\text { Épaisseur } \\
\text { e }\end{array}$ & $\begin{array}{l}\text { Module d'Young } \\
\text { E }\end{array}$ & $\begin{array}{l}\text { Coefficient } \\
\text { de Poisson v }\end{array}$ & $\begin{array}{l}\text { Ressort normal } \\
\mathrm{K}_{\mathrm{n}}\end{array}$ & $\begin{array}{c}\text { Ressort } \\
\text { cisaillement } \mathrm{k}_{\mathrm{s}}\end{array}$ & $\begin{array}{c}\text { Angle } \\
\text { de frottement } \\
\varphi_{i}\end{array}$ \\
\hline Valeurs & $0,14 \mathrm{~m}$ & $2,5.10^{4} \mathrm{MPa}$ & 0,2 & $6.10^{9} \mathrm{~N} / \mathrm{m}^{3}$ & $6.10^{9} \mathrm{~N} / \mathrm{m}^{3}$ & 27 degrés \\
\hline
\end{tabular}

\section{Initialisation des contraintes}

La prise en compte de l'état initial des contraintes dans le sol, exerçant une influence importante sur la réponse de l'ouvrage aux sollicitations qu'on lui applique, constitue un des aspects essentiels de la modélisation géotechnique.

Le mur en Terre Armée expérimental a été édifié en une dizaine d'étapes pendant lesquelles le sol de remblai est mis en œuvre par couches successives de $37,5 \mathrm{~cm}$ d'épaisseur et compacté, et les éléments de renforcement (écailles + armatures) positionnés en phase avec le sol.

L'état de contrainte en fin de construction est le résultat de toutes les sollicitations qu'a subi le sol de remblai lors de la montée du mur. Dans un souci de simplification, la modélisation du phasage des travaux n'a pas été entreprise, mais un état de contrainte proche de celui in situ a cependant été recherché. Dans ce but, l'effet du compactage a été représenté en prenant en compte la surconsolidation subie par les sols à un moment de leur histoire. En première approche, un matériau de remblai compacté avec un objectif Q4, peut être considéré comme un sol surconsolidé avec une contrainte de préconsolidation $\sigma_{p}^{\prime}$ de l'ordre de $300 \mathrm{kPa}$ (Boutonnier et al., 2005). Les contraintes verticales sont estimées en imposant en chaque point du modèle la contrainte engendrée par le poids des terrains sus-jacents. Les contraintes horizontales sont déduites à partir d'une relation empirique pour le calcul du coefficient de pression des terres au repos $\mathrm{K}_{0^{\prime}}$ pour un massif de sol horizontal, surconsolidé (Mayne et Kulhawy, 1982) :

$$
K_{0}=\left(1-\sin \varphi^{\prime}\right) \sqrt{\frac{\sigma_{p}^{\prime}}{\sigma_{v_{0}}^{\prime}}}
$$

où $\sigma_{p}^{\prime}$ est la pression de préconsolidation du sol et $\sigma_{v 0^{\prime}}$ la contrainte verticale effective en place.

Toutefois, pour les couches de sol ayant subi une forte surconsolidation (terrains superficiels) le calcul du coefficient $K_{0}$ est borné par le coefficient de butée des sols $K_{p}$ :

$$
K_{p}=\tan ^{2}\left(\frac{\pi}{4}+\frac{\varphi_{\text {sol }}}{2}\right)
$$

Une fois les contraintes initialisées en prenant en compte la surconsolidation des sols mis en œuvre, l'ouvrage est amené à un état d'équilibre en relâchant progressivement le déplacement du parement : l'état ( Fin de construction ) est alors atteint.

\section{Conditions limites}

Au niveau de la base du sol de remblai, la fosse a été modélisée en partie afin de reproduire l'accroche avec le sol sans pour autant fixer les nœuds à la base du remblai et au pied du mur.

La face inférieure de la fosse est bloquée en déplacement dans les directions $\mathrm{x}, \mathrm{y}$ et $\mathrm{z}$.

Les faces latérales de l'ouvrage, en contact avec les bords de la fosse dans le plot expérimental, sont uniquement bloquées en déplacements suivant la direction longitudinale. La résistance en cisaillement 
apportée à l'interface fosse/ouvrage, dans la direction transversale, n'est donc pas simulée.

\section{9}

\section{Application de la surcharge}

Lors de l'expérimentation, les chargements ont été appliqués sur des traverses en béton, mais ces dernières n'ont pas été représentées dans le modèle numérique. Les surcharges sont directement appliquées au niveau du ballast sous forme de contraintes sur une surface équivalente à la surface d'appui d'une traverse. La masse des traverses, estimée à $m=500 \mathrm{~kg}$, est prise en compte comme une surcharge additionnelle.

La contrainte correspondant à la charge de $90 \mathrm{kN}$ est appliquée, en tête de l'ouvrage, de manière progressive dans le but d'assurer la condition de quasistaticité du chargement et donc du comportement de l'ouvrage. Ainsi, le chargement de $90 \mathrm{kN}$ est imposé de manière incrémentale en 60 paliers, avec atteinte de l'état d'équilibre de l'ouvrage systématique.

\section{0}

\section{Résultats}

L'instrumentation mise en place sur l'ouvrage Terre Armée permet d'atteindre un certain nombre de grandeurs comme les déplacements horizontaux des écailles du parement, le tassement en tête de l'ouvrage, les tractions dans les différents lits d'armatures, de façon à appréhender le comportement de l'ouvrage sous la surcharge de 9 tonnes. Les résultats issus de l'expérimentation peuvent être alors comparés à ceux obtenus via le modèle numérique élaboré.

\section{- Tassements et déplacements du parement}

Les tassements obtenus au niveau de l'interface sous-couche/sol de remblai, à l'aplomb des traverses, et les déplacements horizontaux du parement pour une surcharge de $90 \mathrm{kN}$, ont été assez largement surévalués (Tableau III).

Tabiau II Comparaison des valeurs de tassement et de déplacement horizontal du parement pour les approches expérimentale et numérique. Settlements and horizontal facing displacements for both experimental and numerical approaches.

\begin{tabular}{|c|c|c|}
\hline & 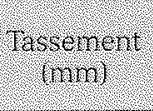 & $\begin{array}{l}\text { Weplagernent } \\
\text { pargnent }\end{array}$ \\
\hline Expérimentation & $\approx 0,1$ & $\approx 0,01$ \\
\hline Modélisation & $\approx 0,5$ & $\approx 0,05$ \\
\hline
\end{tabular}

Compte tenu des dimensions du mur, les déplacements observés sont très faibles, l'ouvrage est finalement peu sollicité et son comportement est par conséquent majoritairement élastique.

Le module élastique imposé a été déduit d'essais de caractérisation établis pour des gammes de déformations probablement beaucoup plus grandes. Le module d'Young à considérer pour une telle sollicitation serait probablement nettement supérieur à celui imposé pour le concours de prévisions, ce qui peut justifier en partie les écarts importants (d'un ordre 5) entre les approches expérimentale et numérique.

\section{- Traction dans les armatures}

Parmi les différents lits d'armatures instrumentés, seuls les résultats concernant le comportement des armatures des lits $1(\mathrm{z}=3,375 \mathrm{~m})$ et $3(\mathrm{z}=1,875 \mathrm{~m})$ sont présentés par la suite; le lit 1 (resp. 3) étant situé dans la partie supérieure (resp. inférieure) du mur.

Les incréments de tractions étant faibles (quelques \% seulement) par rapport aux tractions réelles dans les armatures, l'état de fin de construction coïncide presque avec celui de fin de chargement.

Le modèle numérique présenté, caractérisé par ses paramètres mécaniques, l'initialisation des contraintes et la mise en équilibre de l'ouvrage, affiche une certaine tendance à sous-estimer les efforts de tractions dans les armatures (Fig. 6). Toutefois, l'ordre de grandeur et l'évolution des incréments de traction lors du chargement sont assez bien représentés, qu'il s'agisse de l'armature superficielle (lit 1) qui présente une zone de relâchement des tractions bien identifiée, ou de l'armature plus enfouie (lit 3) dont l'incrément est plus monotone.

\section{Étude paramétrique}

A posteriori, des essais d'arrachement ont été menés sur les armatures en place dans l'ouvrage (Soyez et Bourgeois, 2009). La simulation de ces essais permet par analyse inverse d'aboutir à des valeurs de raideur et de frottement d'interface $\left(\varphi_{\text {exp }}\right)$ directement calées à partir de l'approche expérimentale (Tableau IV).

\begin{tabular}{|c|c|c|}
\hline TABLEAU WV & \multicolumn{2}{|c|}{$\begin{array}{l}\text { Modification des paramètres caractérisant } \\
\text { l'interface armature/sol à partir de la simula- } \\
\text { tion d'essais d'arrachement. } \\
\text { Strip/soil interface parameters estimated from pull } \\
\text { tests. }\end{array}$} \\
\hline Anterface & Waracurt r & 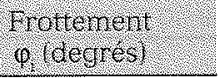 \\
\hline $\begin{array}{c}\text { Estimation } \\
\text { préliminaire }\end{array}$ & $10^{6} \mathrm{~N} / \mathrm{m}^{2}$ & 27 \\
\hline $\begin{array}{c}\text { Calage } \\
\text { expérimental }\end{array}$ & $4.10^{6} \mathrm{~N} / \mathrm{m}^{2}$ & $\begin{array}{c}64 \text { à } 81 \\
\left(\varphi_{\mathrm{exp}}\right) \\
\end{array}$ \\
\hline
\end{tabular}

L'approche qui avait permis d'estimer la raideur d'interface repose sur l'analyse du déplacement apparent $d_{a p p}$ d'une armature pour atteindre la résistance maximale au niveau d'une armature. Or le déplacement apparent peut être traduit par la composée du déplacement au niveau de l'interface $d_{i^{\prime}}$ et du déplacement $d_{s}$ dû à la déformation du sol environnant.

Le fait que la raideur d'interface rend compte seulement du comportement à l'interface, explique une partie de la sous-estimation de ce paramètre en première approche.

Initialement, seul le frottement résiduel $\left(\tan \varphi_{i}=\right.$ $0,7 \times \tan \varphi_{\text {sol }}$ à l'interface armature/sol a été modélisé. Or les angles de frottement nécessaires pour simuler les essais d'arrachement ont été nettement supérieurs à celui estimé en première approche.

Le phénomène de dilatance empêchée, essentiellement concentré à proximité de l'armature, s'avère com- 
Traction fin de chargement - Lit 1 - $90 \mathrm{kH}$

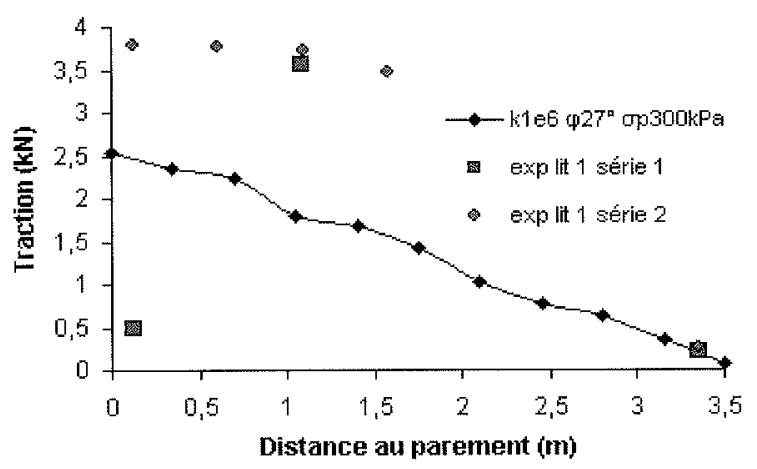

Traction fin de chargement - Lit 3 - $90 \mathrm{kN}$

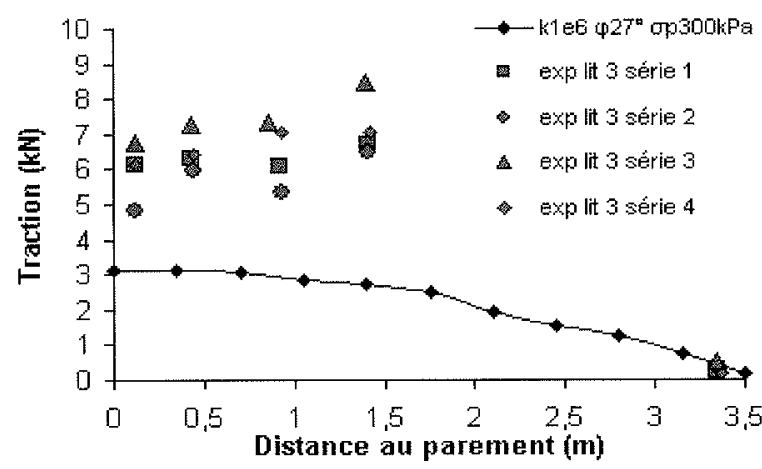

Incrément de traction - Lit 1 - $90 \mathrm{kN}$

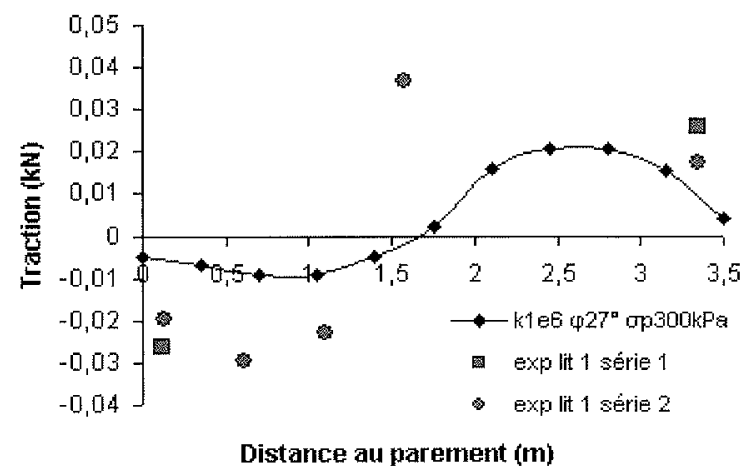

Incrément de traction - Lit $3-90 \mathrm{kN}$

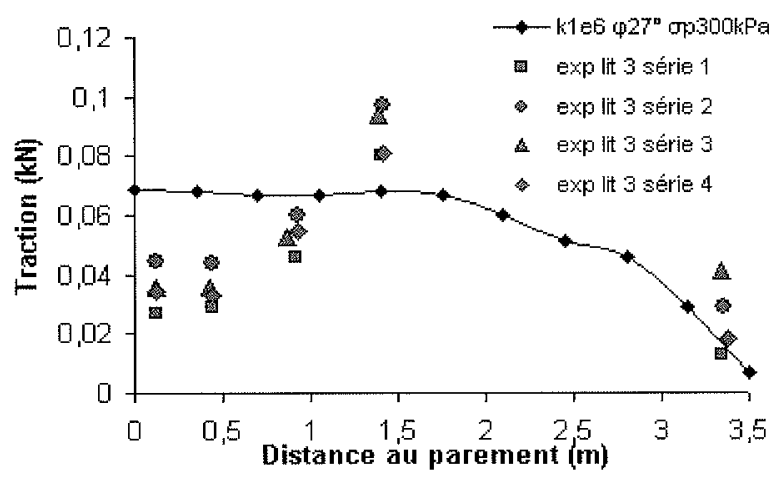

Efforts de traction dans les armatures des lits 1 et 3 et comparaison avec les
Tensile forces at the strip levels 1 and 3 , and comparison with experimental results.

plexe à modéliser localement. Un angle de frottement apparent, englobant le frottement résiduel, phénomène de dilatance empêché et éventuellement une cohésion, est finalement calé à partir des essais d'arrachement.

Les valeurs données dans Abdelouhab et al. (2007), issues d'essais d'arrachement en laboratoire, sont sensiblement inférieures à celles calées expérimentalement. Cependant, pour l'étude du mur en Terre Armée, le compactage des couches engendre un état surconsolidé du sol qui a pour effet d'augmenter significativement le phénomène de dilatance empêchée.

Pour appréhender au mieux le comportement de ce type de mur de soutènement, le calage des paramètres caractérisant l'interface armature/sol, et l'estimation des contraintes initiales dans le sol et les armatures, apparaissent comme des enjeux primordiaux de la modélisation numérique. Leur influence a été mise en évidence lors d'une étude paramétrique dont les valeurs de références pour les armatures sont celles déterminées ci-dessus et l'initialisation des contraintes dans l'ouvrage est menée avec une contrainte de consolidation inférieure à celle déterminée dans le calcul initial (Tableau V).

TubEu v Récapitulatif des valeurs de référence pour chaque paramètre faisant l'objet d'une étude paramétrique.

Reference parameters used for parametric calculations.

\begin{tabular}{|c|c|c|c|}
\hline 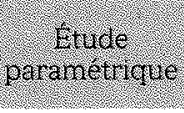 & Rarctedr K & Frottenert & $\begin{array}{l}\text { Contrainte de } \\
\text { consolidation } \\
0 \text { ? }\end{array}$ \\
\hline Référence & $4.10^{6} \mathrm{~N} / \mathrm{m}^{2}$ & $\varphi_{\mathrm{e}}$ & $150 \mathrm{kPa}$ \\
\hline
\end{tabular}

Les graphes de la figure 7 montrent une forte influence de la valeur de l'angle de frottement d'interface pour l'armature du lit 1 tandis qu'elle est quasiment inexistante pour l'ensemble des autres lits d'armature. En effet, pour un frottement apparent faible $\left(\varphi_{i}=27\right.$ degrés) les armatures supérieures (lit 1) sont les seules à voir leur interface plastifier. Toutes les autres interfaces restent dans leur domaine élastique. Ce constat vient justifier la forte dépendance des résultats à la raideur d'interface $\mathrm{k}_{\mathrm{i}}$ (Fig. 8). Ce paramètre caractérisant le domaine élastique de l'interface est difficile à approcher sans résultat d'essais d'arrachement pour se caler, mais ses variations engendrent des écarts importants sur les résultats, notamment pour de faibles sollicitations de l'ouvrage.

L'initialisation des contraintes, en tenant compte du compactage comme un état surconsolidé du sol de remblai, permet d'atteindre des contraintes horizontales plus élevées et des sollicitations des armatures plus importantes que la seule mise en équilibre statique de l'ouvrage ne le permettrait.

Les résultats présentés sur la figure 9 montrent des écarts importants sur les tractions en fonction des contraintes induites initialement dans le sol de remblai. La connaissance de l'état initial de l'ensemble sol + renforcement semble être primordiale pour le calcul du comportement de la structure sous chargement.

En pratique, la simple génération de surcontraintes dans l'ouvrage permet seulement d'aboutir à une approximation de l'état de la structure en fin de construction. Idéalement, il serait envisagé de modéliser l'ensemble du phasage de construction, en prenant des lois de comportement capable de prendre en compte la consolidation des sols sous compactage. 

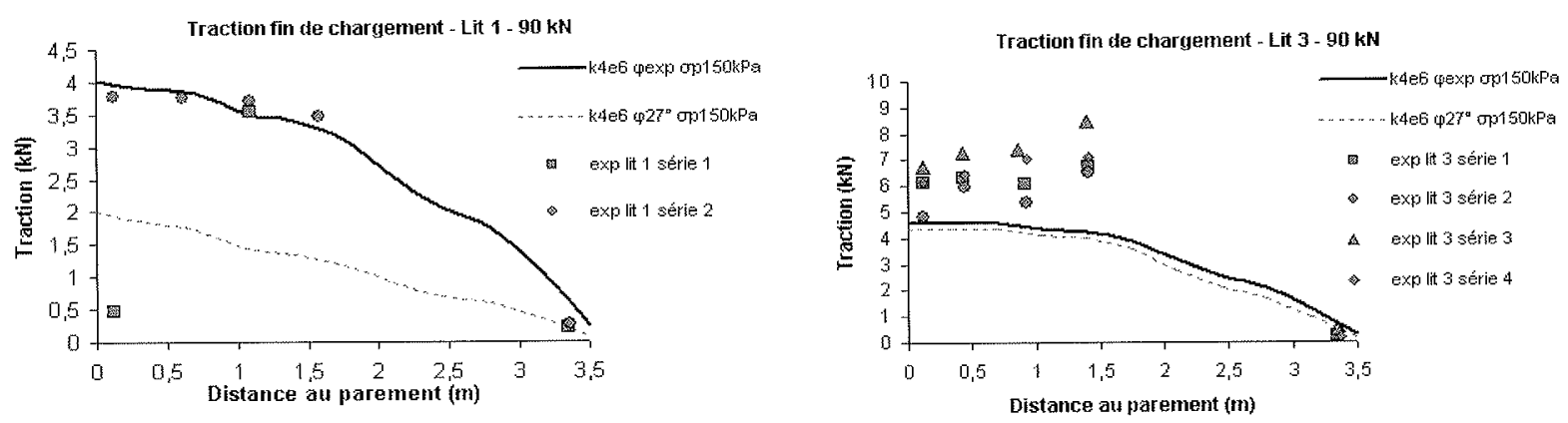

HG. 7 Influence du frottement d'interface sur les efforts de traction des lits 1 et 3. Interface friction influence on tensile forces at the strip levels 1 and 3.
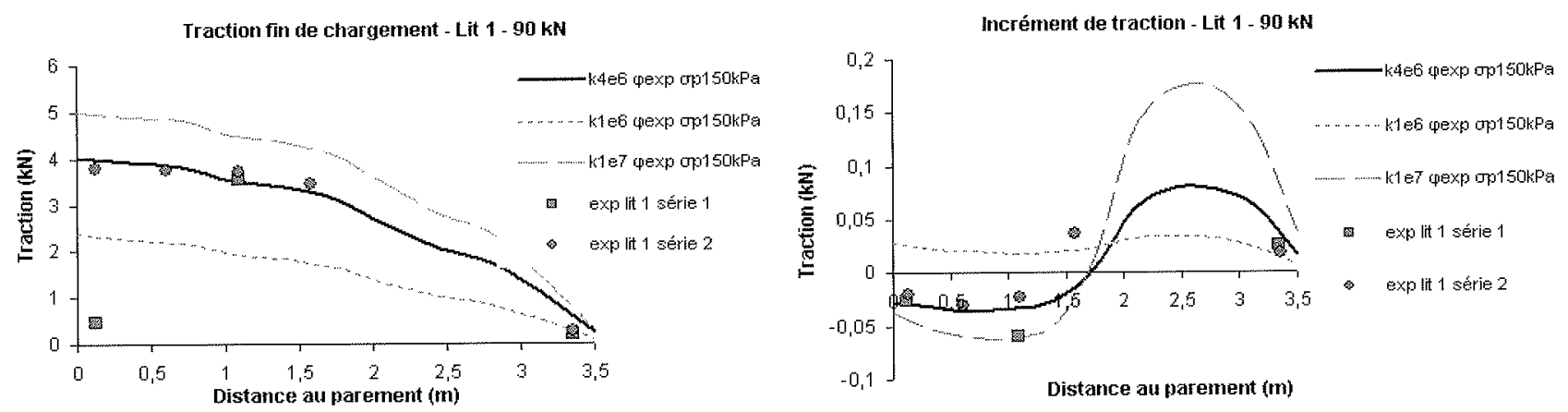

HG. 8 Influence de la raideur d'interface sur les efforts de traction du lit 1. Interface stiffness influence on tensile forces at the strip level 1.
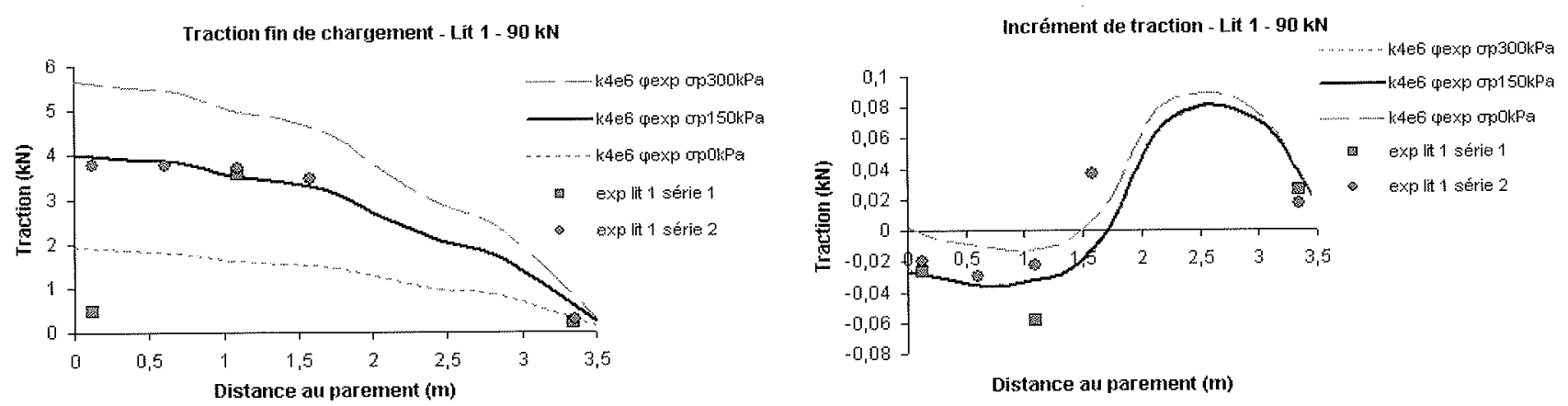

FG. 9 Influence de la contrainte de préconsolidation des sols lors de leur mise en place, sur les tractions du lit 1. Influence of preconsolidation stress, affected during the stress initialisation, on tensile forces at the strip level 1.

\section{2}

\section{Conclusion}

Le concours de prévisions organisé sur l'étude d'un mur en Terre Armée a représenté une opportunité intéressante de confronter le comportement de l'ouvrage réel avec celui prédit par des approches numériques.

Les modèles $3 \mathrm{D}$, avec la modélisation de chaque élément de renforcement (armature + écailles) et de leur interaction avec le sol environnant, permettent d'accéder à une estimation plutôt réaliste du comportement, à condition de disposer des caractéristiques des matériaux correspondant aux gammes de sollicitation de l'ouvrage, et d'un certain nombre d'essais (arrachement par exemple) pour ajuster le calage des paramètres mécaniques.

En un sens, une des plus grandes difficultés de la modélisation numérique réside en la mise en place d'une procédure permettant d'aboutir fidèlement à l'état mécanique de l'ouvrage en fin de construction.

La sollicitation appliquée s'étant finalement avérée être faible devant la résistance mécanique de l'ouvrage, le comportement de l'ouvrage et de ses composants est en grande majorité élastique. Sortir de ce domaine, via des chargements plus importants, aurait pu permettre de confronter le calage des paramètres dans le domaine plastique. 
Abdelouhab A., Dias D., Bouredeau Y., Freitag N. - Soil/reinforcement interface characterization using three-dimensional physical modelling. The 5th International Symposium on Earth Reinforcement «IS Kyushu 2007 », Fukuoka, Japan, 2007.

AFNOR - Renforcements des sols. Ouvrage en sols rapportés renforcés par armatures ou nappes peu extensibles. Norme française NF P 94-220-0, 1998.

Bolton M.D. - The strength and dilatancy of sands. Geotechnique 36 (1), 1986 p. 65-78.
Boutonnier L., Guerpillon Y., Flavigny E., Barnichon J.D. - Réutilisation des matériaux de dépôts anciens en remblai. TREMTI (Traitement et Retraitement des Matériaux pour Travaux d'Infrastructures), $2^{e}$ Symposium international, Paris, 2005.

Ingold T.S. - Lateral earth pressures induced by compaction. International conference on compaction, Paris 22-23-24 avril 1980, volume I, Éditions Anciens ENPC, p. $145-150$

Itasca consulting group 2006. FLAC3D 3.1 Structural Elements.
Mayne P.W., Kulhawy F.H. - K0-OCR relationships in soils. ASCE, Journal of geotechnical engineering division, vol. 12, 1 , 1982, p. 142-146.

Mestat P. - État de contraintes initiales dans les sols et calcul par éléments finis. Bulletin des laboratoires des ponts et chaussées, 215, mai-juin 1998, p. 15-32.

Soyez L., Bourgeois E. - Étude du com portement d'un mur en Terre Armée : rôle de l'interface sol/armature dans la modélisation, Revue française de géotechnique, $\mathrm{n}^{\circ} 129,2009$ 